\title{
AuNPs for identification of molecular signatures of resistance
}

\author{
Bruno Veigas ${ }^{1,2}$, Alexandra R. Fernandes ${ }^{3}$ and Pedro V. Baptista ${ }^{1}$ * \\ ' Nanotheranostics, Centro de Investigação em Genética Molecular Humana, Departamento de Ciências da Vida, Faculdade de Ciências e Tecnologia, \\ Universidade Nova de Lisboa, Caparica, Portugal \\ ${ }^{2}$ Centro de Investigação em Materiais, Departamento de Ciências de Materiais, Faculdade de Ciências e Tecnologia, Universidade Nova de Lisboa, Caparica, \\ Portugal \\ ${ }^{3}$ Centro Química Estrutural, Departamento de Ciências da Vida, Faculdade de Ciências e Tecnologia, Universidade Nova de Lisboa, Caparica, Portugal
}

\section{Edited by:}

Marta Martins, University College

Dublin, Ireland

\section{Reviewed by:}

Lilia Macovei, The Forsyth Institute, USA

Noton Kumar Dutta, Johns Hopkins University, USA

\section{${ }^{*}$ Correspondence:}

Pedro V. Baptista, Nanotheranostics, Centro de Investigação em Genética Molecular Humana, Departamento de Ciências da Vida, Faculdade de Ciências e Tecnologia, Universidade Nova de Lisboa, Campus de Caparica, 2829-516 Caparica, Portugal

e-mail: pmvb@fct.unl.pt
The increasing levels of drug resistance are one of biggest threats to overcome microbial infection. The ability to rapidly and accurately detect a given pathogen and its drug resistance profile is essential for the appropriate treatment of patients and for preventing further spread of drug-resistant strains. The predictive and informative value of these molecular markers needs to be translated into robust surveillance tools that correlate to the target and extent of resistance, monitor multiresistance and provide real time assessment at point-of-need. Rapid molecular assays for the detection of drug-resistance signatures in clinical specimens are based on the detection of specific nucleotide sequences and/or mutations within pre-selected biomarkers in the genome, indicative of the presence of the pathogen and/or associated with drug resistance. DNA and/or RNA based assays offer advantages over phenotypic assays, such as specificity and time from collection to result. Nanotechnology has provided new and robust tools for the detection of pathogens and more crucially to the fast and sensitive characterisation of molecular signatures of drug resistance. Amongst the plethora of nanotechnology based approaches, gold nanoparticles have prompt for the development of new strategies and platforms capable to provide valuable data at point-of-need with increased versatility but reduced costs. Gold nanoparticles, due to their unique spectral, optical and electrochemical properties, are one of the most widely used nanotechnology systems for molecular diagnostics. This review will focus on the use of gold nanoparticles for screening molecular signatures of drug resistance that have been reported thus far, and provide a critical evaluation of current and future developments of these technologies assisting pathogen identification and characterisation.

Keywords: nanotechnology, nanodiagnostics, gold nanoparticles, AuNPs, multidrug resistance, Tuberculosis, molecular diagnostics technologies

\section{THE CASE FOR MOLECULAR CHARACTERISATION OF PATHOGENS}

Rapid and specific detection and characterisation of agents involved in infection is of paramount importance to deliver successful treatment. Traditional methodologies for identification of pathogens, though of extreme relevance, may be laborious and time-consuming, which may lead to delayed definitive diagnoses and treatment to the patient (Mothershed and Whitney, 2005). Several innovative approaches have already made their way to the clinics and provide for increased sensitivity and specificity pathogen detection and characterisation and doing so in a fast multiplexed (Hauck et al., 2010). Additionally, the literature is full of new concepts for nucleic acid-based tests (NATs) for bacteria detection that may still make their way to the clinical setting.

Nucleic acid-based tests can be used to detect the presence of organisms directly in clinical specimens without the need of culture. In addition, hospital infection control and epidemiology programs are benefiting from the use of NATs for detecting antibiotic resistance genes and for subtyping bacteria.
The first NAT cleared for use by the Food and Drug Administration (FDA) was the Gen-Probe PACE test (1988) that used nucleic acid hybridisation to detect Chlamydia sp. and Gonococci. Introduction of PCR allowed the development of a plethora of diagnostic approaches for clinically relevant bacterial pathogens (Mothershed and Whitney, 2005). One such example, already in the market, is the line probe assay (LiPA) from Innogenetics (Gent, Belgium). Innogenetics produces several line probe NATs for bacterial detection including ones for Mycobacterium tuberculosis complex and Mycobacterium spp., rpoB gene mutations conferring rifampicin resistance, and Treponema pallidum antibodies. The INNO-LiPA Rif. TB test detects the M. tuberculosis complex (MTBC), specifically five genotypes corresponding to sensitivity to rifampicin and four resistant genotypes. While there is great potential of molecular assays to increase the speed and accuracy of bacterial identification in the clinical laboratory, limitations of NATs must be considered. For example, sample preparation and DNA purification from complex media constitutes a serious drawback for these assays since quantity and quality of template/target is one of the main aspects that 
affect performance. Also, costs associated to specialized training for personal and sophisticated equipment pose a serious obstacle for the widespread implementation of NATs as front line diagnostics.

\section{NANOTECHNOLOGY FOR MOLECULAR DIAGNOSTICS (NANODIAGNOSTICS)}

In the last decade, the use of nanomaterials for biosensing has been having a great impact and presents a great opportunity to develop fast, accurate and cost effective approaches for detection of pathogenic infectious agents. Nanodiagnostics have focused on the design of systems where researchers manipulate the properties of nanostructures for diagnostics purposes. Compared to standard methodologies, nanotechnology based approaches have several important practical advantages, including: enhanced surface reactivity, quantum confinement effects, enhanced electrical conductivity and enhanced magnetic properties, which enable nucleic acid detection to be extremely sensitive (Kaittanis etal., 2010; Chi et al., 2012; Shinde et al., 2012; Hartman et al., 2013). It should be mentioned that several systems incorporating small peptide and/or protein recognition moieties have also been reported, but fall outside the scope of the present review (for additional insights please refer to Larguinho and Baptista, 2012).

Despite the wide range of nanoscale systems for biomolecular assays (Azzazy etal., 2006; Jain, 2007; Das et al., 2010), the most promising approaches are based on nanoparticles (NPs; Rosi and Mirkin, 2005; Jain, 2007; Baptista et al., 2008; Branton et al., 2008; Tallury et al., 2009; Jung et al., 2010; Chi etal., 2012; Wang etal., 2013; Lin etal., 2014). In particular, the unique properties of noble metal NPs, such as gold, have allowed for the development of new biosensing platforms, offering greater sensitivity than conventional reporter molecules (Azzazy and Mansour, 2009). Surface chemistries of AuNPs can be easily tuned and functionalised with organic thiol molecules or thiol-containing polymers, leading to the formation of relatively strong covalent bonds (Kaittanis et al., 2010). For example, gold nanoparticles (AuNPs) conjugated with specific oligonucleotides can sense complementary DNA strands in a nearly one-on-one interaction between the NP and the target DNA molecule (Baptista et al., 2005; Jain, 2005; Azzazy et al., 2006, 2007; Veigas et al., 2012a). AuNPs' simplicity and versatility have attracted considerable attention towards the development of molecular diagnostic applications and are becoming a critical component of nanotechnology-based detection of pathogens (Liu, 2006). AuNPs support multiple detection platforms, i.e., a target analyte can be sensed through more than one detection methodology, such as spectroscopic, colorimetric, fluorimetric and electrochemical methods (Jung et al., 2010; Upadhyayula, 2012).

Gold nanoparticles have unique optical properties associated with a well-defined surface plasmon resonance (SPR) band in the visible region of the spectrum (Halfpenny and Wright, 2010), strongly correlated to composition, shape and inter-particle distance (Johnson etal., 2007). For example, AuNP aggregation leads to a pronounced color transition from red to blue due to plasmon coupling between NPs (Jain, 2007). Consequently, most AuNPs based methods rely on these colorimetric changes of the colloidal solution upon aggregation derived from changes to the media dielectric and/or due to recognition of a specific target. These detection strategies typically depend on the interaction between nanostructure-bound oligonucleotides and the target molecule mediated by a recognition element, which, for DNA/RNA assays, is an oligonucleotide sequence - gold nanoprobe (Au-nanoprobe). A specific complementary target may hybridize to the Au-nanoprobes and promote inter-particle cross-linking aggregation (e.g., using two Au-nanoprobes each functionalised with one half of a contiguous target recognition sequence) or stabilize nanoprobes against changes to the media dielectric (e.g., pH, ionic strength). In the latter, hybridisation of $\mathrm{Au}$-nanoprobes to the target sequence will prevent the non-crosslinking aggregation induced by increasing ionic strength (Figure 1; Sato et al., 2003; Baptista et al., 2005). Thus, modulation of AuNP or Au-nanoprobe inter-particle distance allows control over their corresponding aggregation and dispersion levels providing visual detection for a wide range of biological entities (Hauck et al., 2010; Ngo et al., 2011).

\section{NANODIAGNOSTICS FOR PATHOGENS}

Identification of pathogens based on specific target sequences has become the corner-stone of molecular based approaches to discriminate between organisms and characterize particular variations at the genomic level that provide unique nucleic acid signatures suitable for diagnostics. This trend has had a new surge with the development of numerous nanoparticle-based approaches designed to identify those pathogen signatures with extra sensitivity and faster than ever before (Costa et al., 2010; Kaittanis et al., 2010; Chi et al., 2012; Veigas et al., 2012a). In fact, the increase in sensitivity without loss of selectivity and specificity has promoted nanodiagnostics in the field of pathogen characterisation. Also, due to the minute dimensions of the signal transduction label (e.g., AuNPs), these systems show a high degree of miniaturization that makes them suitable for use at point-of-care or point-of-need.

The first proof-of-concept for nanodiagnostics using AuNPs was introduced by Mirkin et al. (1996) who successfully demonstrated that $13 \mathrm{~nm}$ AuNPs functionalized with a specific oligonucleotide sequence selectively assembled in the presence of a complementary target DNA. The development of this proof-ofconcept led to the development of the first Au-nanoprobe based pathogen diagnostic system, allowing the detection of anthrax via a specific lethal factor DNA sequence (Bailey et al., 2003). This approach was further explored in a multitude of targets and samples (for further insights see Kaittanis et al., 2010; Veigas et al., 2012a and references therein).

Following a similar technological approach, Baptista et al. (2006) developed a rapid and relatively low cost method for DNA detection and generated the first application of AuNPs for the molecular diagnostics of Mycobacterium tuberculosis (Mtb). The method consists in differential stabilization of Aunanoprobes in presence of DNA targets following salt induced aggregation: presence of a complementary target prevents nanoprobe aggregation and the solution remains red; whereas non-complementary/mismatched targets do not prevent gold 
AuNPs Cross-Linking

Approach

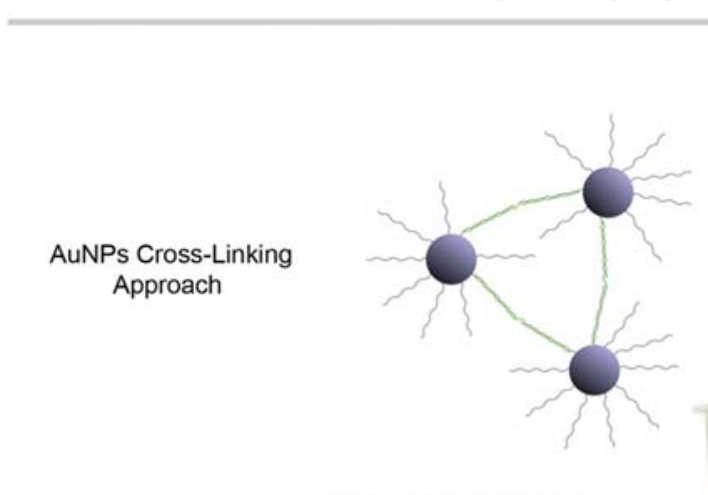

AuNP-Target-AuNP complex

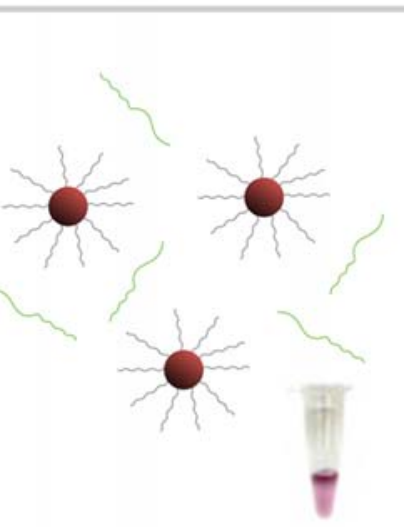

Hybridization induced aggregation

\section{AuNPs non-Cross-Linking Approach}
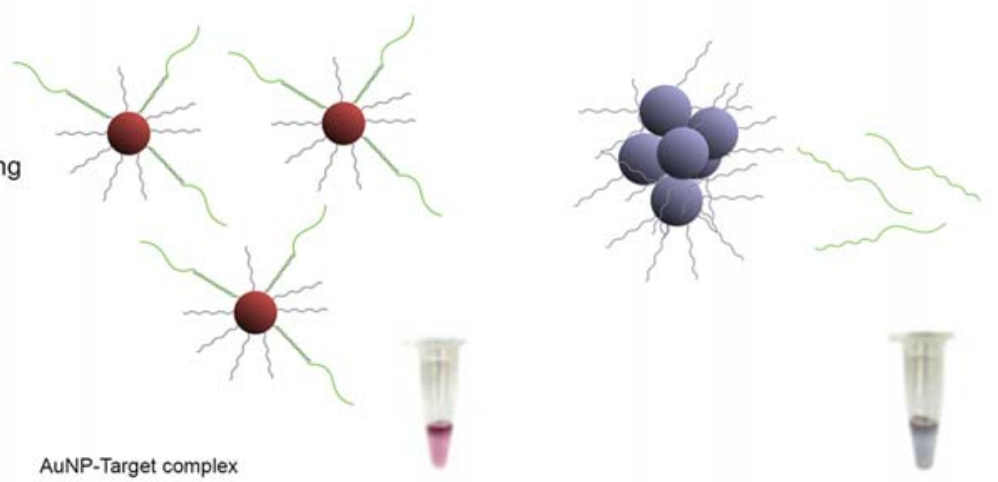

AuNPs - Sandwich based assays

Salt induced aggregation

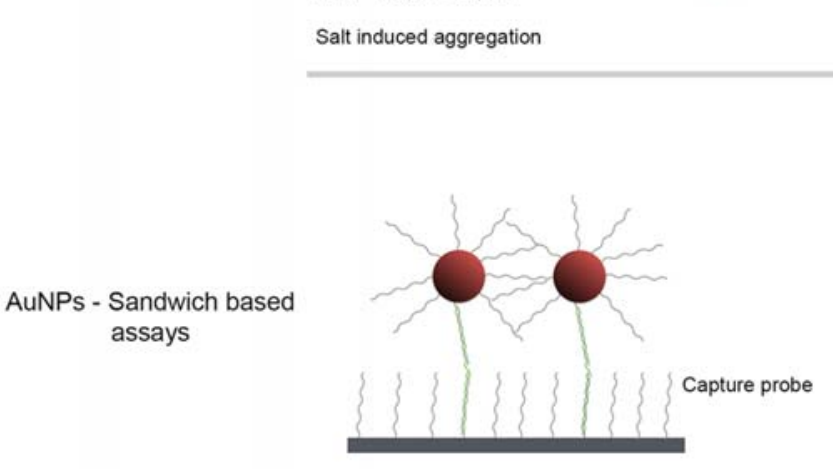

AuNP-Target-Surface complex

Nanoparticles as signal enhancers
FIGURE 1 | AuNP detection schemes for characterization of antibiotic resistant pathogen strains. Detection strategies based on the observable colorimetric alteration of Au-nanoprobes solutions. The surface plasmon band (SPR) of gold nanoparticles depends on inter-particle distance, size and aggregation. Aggregation of AuNPs results in a red-shift of the SPR with concomitant change of color of the solution, from red to blue.

(A) Cross-Linking method relies on the hybridization of two populations of Au-nanoprobes that bind to adjacent regions of a nucleic acid target. Aggregation mediated by DNA hybridization results in a visual change of color from red to blue. (B) Non-cross-linking method relies on the differential aggregation profiles of Au-nanoprobes induced by increased ionic strength in the presence or absence of the specific target sequence: presence of the complementary target sequence to that of the probe prevents aggregation and the solution remains red, whereas absence of a specific target sequence leads to extensive aggregation after salt addition and the solution turns blue. (C) Sandwich based assay involves an immobilized capture probe, target DNA and one Au-nanoprobe reporter. Half of the target DNA hybridizes to the immobilized DNA and the other half to the reporter Au-nanoprobe. This strategy allows a simple to preform surface wash, thus increasing the signal to noise ratio and consequently the sensitivity. This method enables a higher sensitivity when coupled to an improved hybridization method that facilitates probe-target binding in a homogeneous format. 
nanoprobe aggregation, resulting in a visible change of color from red to blue. The methodology was tested in clinical samples demonstrating high efficiency with results comparable to those attained via commercial molecular tuberculosis (TB) diagnostics test, such as INNO-LiPA Rif. TB (Veigas et al., 2012a). Similar approaches have been used by Liandris et al. (2009) who developed a non-cross-linking approach for the detection of TB without the need of target amplification. Following a cross-linking approach, Soo et al. (2009) designed a set of gold nanoprobes to specifically hybridize with target DNA from Mtb strains. This methodology was evaluated by directly and simultaneously detecting M. tuberculosis complex (MTBC) and Mtb in 600 clinical strains.

Staphylococcus aureus is also one of the most important human pathogens, causing more than 500,000 infections in the US each year (Chang etal., 2013). By using aptamers that specifically recognize $S$. aureus, Chang etal. (2013) developed an ultrasensitive aptamer-conjugatedAuNPs for rapid bacterial detection. Their non-polymerase chain reaction (PCR)-based method measures the resonance light-scattering signal of aptamer-conjugated AuNPs to detect a single cell within $1.5 \mathrm{~h}$. Accordingly to the authors this platform technology has the potential to develop a rapid and sensitive bacterial testing at point-of-care (Chang et al., 2013).

\section{GOLD NANOPARTICLES CHARACTERISATION OF ANTIBIOTIC RESISTANCE PROFILES}

For the past 20 years there has been an increase in the emergence of antibiotic-resistant microorganisms with elevated pathogenesis at the global level, leading to an urgent need for new and improved approaches for bacterial quantification and identification (Pissuwan etal., 2010). Table 1 summarizes existing AuNP-based technologies for the antibiotic susceptibility characterization of pathogens. Particularly problematic drug resistant bacteria include the methicillin-resistant strains of $S$. aureus (MRSA), responsible for many opportunistic infections, enteropathogenic Escherichia coli, Mtb showing multidrug resistance (MDR-TB and XRD-TB), and Streptococcus pneumoniae. The prevalence of drug resistant strains of Mtb (MDR and XDR-TB) and MRSA have demonstrated the need for the development of drug susceptibility systems that are capable of delivering an unequivocal response to identify with high sensitivity and in a cost-efficient manner the pathogen's resistance profile and allowing fast and accurate therapeutic approach (Kaittanis et al., 2010).

New diagnostic tools for drug resistant pathogen detection and characterisation ought to overcome the main constraint in terms of current molecular diagnostics - time. Several new technologies are currently being developed and validated to provide faster and at a low cost diagnosis of resistant pathogens comparing to conventional culture and drug susceptibility tests. Three distinct operational steps that are typically required for pathogen detection and characterization: sample preparation, target amplification, and signal read-out. Although each step can be considered individually, it is important to emphasize that a key challenge for development of such nucleic acid detection methods is the integration of all these steps into a unified process workflow (Figure 2). Rapid and cost effective diagnosis will have several benefits: earlier treatment of patients, reduction of time spent on inappropriate and ineffective treatment (thereby promoting the development of further drug resistance), and reduction of resistant strains spreading in congregate settings (Veigas et al., 2012a).

AuNPs for molecular detection of antibiotic resistance in S. aureus Methicillin-resistant strains of $S$. aureus is responsible for 40$60 \%$ of all S. aureus infections in hospitals in the United

Table 1 | AuNPs-based systems for pathogen antibiotic susceptibility characterization.

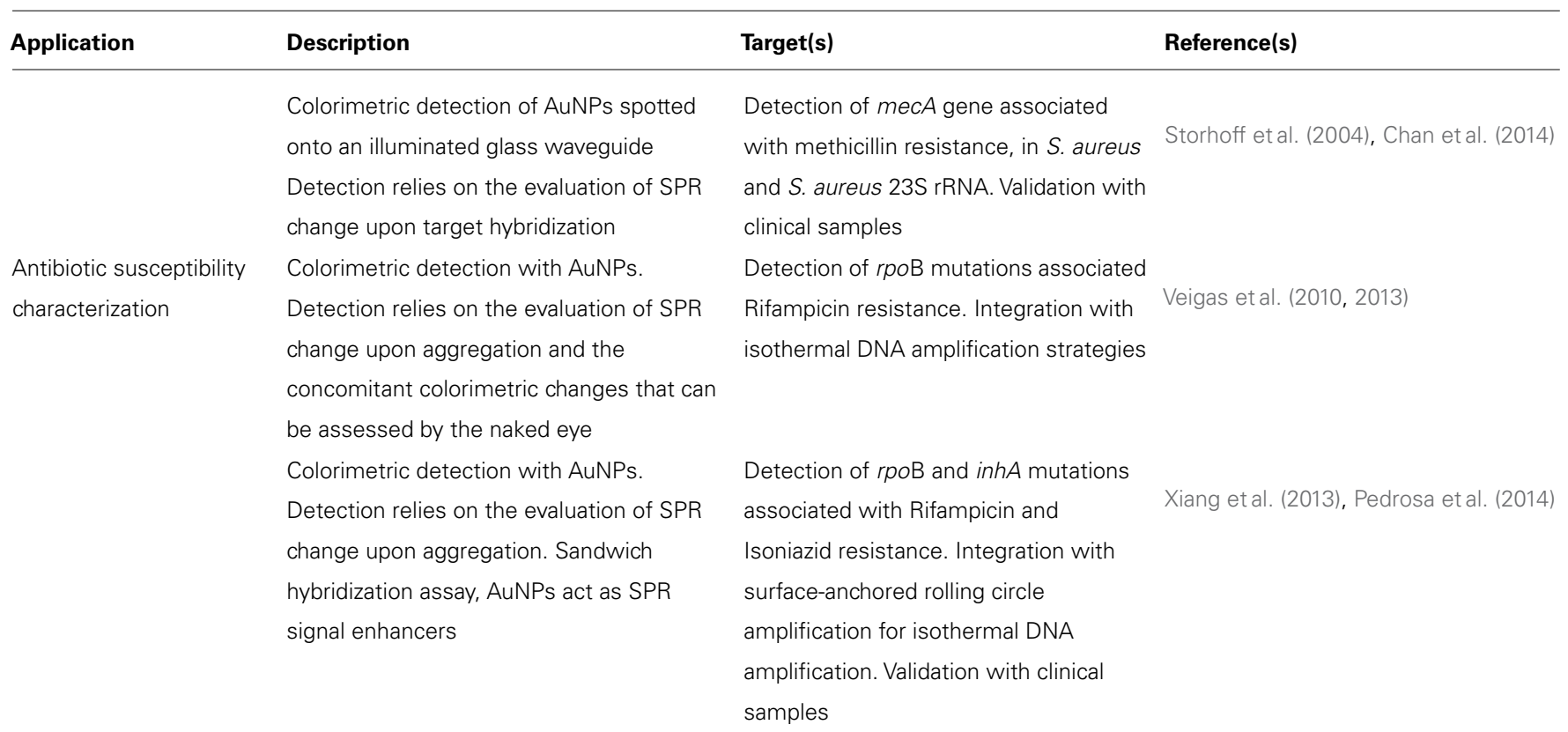




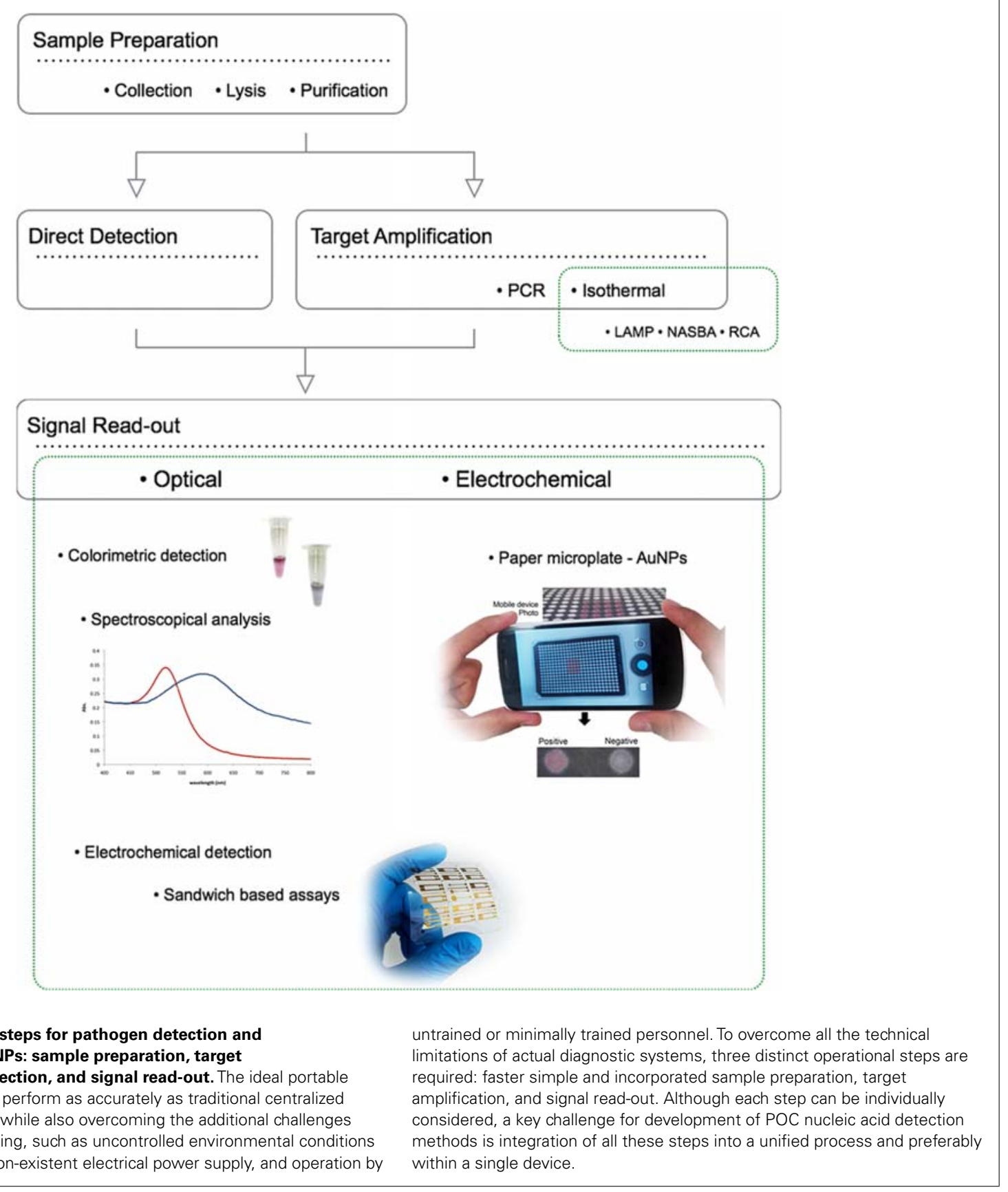

States and United Kingdom (Pelgrift and Friedman, 2013). Currently, the two major strategies for laboratory detection of MRSA are bacterial culture-based phenotypic methods and NATs (Chastre etal., 2014). While a bacterial culture-based phenotypic approach offers the advantages of detecting live bacteria providing antimicrobial susceptibilities and a variety of specimens, they require 1-2 days for the confirmation of MRSA infection, due to their dependence on bacterial growth. In contrast, nucleic acid detection assays allow rapid and sensitive detection of MRSA-specific sequences directly from clinical specimens with a turnaround time ranging from 1 to $3 \mathrm{~h}$ (Bischof etal., 2009; Arbefeville etal., 2011; Kelley et al., 2011;
Chadwick et al., 2013; Chastre et al., 2014). Combining NATs and AuNPs in a single system allows the identification the pathogen's resistance profile with high sensitivity and in a cost-efficient manner providing fast and accurate therapeutic approaches (see Table 2).

In this case, Storhoff et al. (2004) proposed the use of AuNPs for the colorimetric detection of antibiotic resistant $S$. aureus strains back in 2004 via a colorimetric "spot- and-read" assay for the detection of mecA in MRSA genomic DNA samples. In this assay, nucleic acid targets are recognized by DNA-modified Au-nanoprobes that undergo a color change that is visually detectable when solutions are spotted onto an illuminated glass waveguide. This scatter-based 
Table 2 | AuNPs-based systems for pathogen antibiotic susceptibility characterization, preclinical/clinical metadata of sensitivity and specificity.

\begin{tabular}{|c|c|c|c|c|c|}
\hline Pathogen & Target & Sensitivity & Specificity & $\begin{array}{l}\text { No. of } \\
\text { isolates }\end{array}$ & Reference \\
\hline \multirow[t]{2}{*}{ MDRTB } & $\begin{array}{l}\text { Detection of rpoB mutations } \\
\text { associated Rifampicin resistance }\end{array}$ & $\begin{array}{l}84.7 \% \text { (compared to NNO-LiPA Rif. } \\
\text { TB assay) }\end{array}$ & $\begin{array}{l}\text { 100\% (compared to } \\
\text { NNO-LiPA Rif. TB } \\
\text { assay) }\end{array}$ & 46 & Veigas et al. (2010) \\
\hline & $\begin{array}{l}\text { Detection of rpoB and inhA } \\
\text { mutations associated with } \\
\text { Rifampicin and Isoniazid resistance. } \\
\text { Integration with surface-anchored } \\
\text { rolling circle amplification for } \\
\text { isothermal DNA amplification. } \\
\text { Validation with clinical samples }\end{array}$ & $\begin{array}{l}\text { Analitycal sensitivity of } 8.2 \mathrm{pg} \mathrm{uL}^{-1} \\
\text { of genomic DNA from clinical } \\
\text { samples }\end{array}$ & n.d. & 5 & Xiang et al. (2013) \\
\hline
\end{tabular}

method enabled the detection of nucleic acids while demonstrating a remarkable sequence specificity that allowed discrimination of single-base mismatches, deletions or insertions (Storhoff et al., 2004). The method relies on the cross-linking approach targeting the bacterial mecA gene with a limit of detection of $33 \mathrm{nM}$. This approach was effective in discriminating genomic DNA samples of MRSA from methicillin-sensitive S. aureus (MSSA) strains, where the detectable color change was observed only for MRSA with very short hybridisation times (Storhoff et al., 2004). The use of scatter light analysis coupled to the molecular identification approach greatly enhanced detection sensitivity ( $\sim 4$ orders of magnitude) compared to previously reported absorbancebased spot test, thus enabling detection of zeptomole amounts of DNA target. This sensitivity is possible in a homogeneous format because aggregate formation is detectable even when only a very small fraction of the nanoparticle probes is involved in the hybridisation, suggesting a large change to both color and intensity of scattered light from the complexes (Storhoff et al., 2004).

Recently, Chan etal. (2014) reported the use of AuNPs for direct colorimetric PCR detection of MRSA in clinical specimens. The colorimetric assay comprised probes functionalised with specific oligonucleotides targeting $S$. aureus $23 S$ rRNA and mecA sequences. In this study, 72 clinical samples were tested, including positive blood culture, urine, respiratory samples, as well as wound swabs, pus and body fluid. Using conventional bacterial culture as gold standard, the sensitivity, specificity, positive and negative predictive values of this colorimetric assay were 97.14, 91.89, 91.89, and $97.14 \%$, respectively. This performance compares to that of commercial real-time PCR assays but at lower cost per reaction. The colorimetric assay also demonstrated very good agreement with the "gold standard" (94.44\%). This study was the first report on the use of AuNPs colorimetric assay for direct detection of MRSA in various types of clinical specimens (Chan et al., 2014). 
Further evaluation of these assays in large-scale trials is needed which can also allow for some modifications to streamline the procedures for routine use.

\section{AuNPs for molecular characterisation of antibiotic resistance in M. tuberculosis}

Tuberculosis is one of the leading causes of infection in humans, causing high morbility and mortality all over the world. At present, the treatment of choice for an active TB infection is long-term antibiotic therapy, with an initial "intensive phase" consisting of the four first-line anti-TB drugs (isoniazid, INH; rifampicin, RIF; ethambutol, ETH; and pyrazinamide) followed by a typical four month course of RIF and INH alone (Gaspar et al., 2008). Despite effective treatment, due to the length of antibiotic therapy, side effects frequently develop and the associated cost is high (Garner et al., 2007; Aspler et al., 2008; Armstead and Li, 2011). These factors correlate to low patient compliance and contribute to the development of drug-resistant bacteria (Armstead et al., 2011). The rate of new cases of multidrug resistant tuberculosis (MDRTB) continues to increase, and due to the difficulty in the management of such infection, it constitutes a serious health problem (World Health Organization [WHO], 2012). The surge of MDRTB has raised awareness towards extreme resistant TB (XRDTB) or even totally resistant TB. In most cases, drug resistance in Mtb has been related to mutations in several loci within the pathogen's genome. The development of fast, cheap and simple screening methodologies is of paramount relevance for the early detection of these mutations, essential for the timely and effective diagnosis and management of MDRTB patients (Barnard et al., 2008; Veigas et al., 2010; Abebe et al., 2011). Resistance to RIF is commonly associated with point mutations within the $r p o B$ gene of $\mathrm{Mtb}$ whose detection is considered the best early molecular predictor for MDRTB. Resistance to RIF has been associated to single point alterations within a well-defined $81 \mathrm{bp}$ region (codons 507-533) of the $r p o B$ gene encoding for the beta subunit of RNA polymerase. Concurrent resistance to INH and RIF is commonly associated with point mutations in $k a t G$, inh $A$, and rpoB genes of MTBC (Musser, 1995; Soini and Musser, 2001). Prompt diagnosis of MDRTB has been the main obstacle to its correct management and control. This problem would seem to have been solved with the development of molecular techniques applicable also in high-prevalence, low-income settings, such as the Genotype MTBDR- Plus and Gene Xpert MTB/RIF assays. However, though very rapid and highly sensitive, these tests are not considered highly specific for the diagnosis of RIF resistance, particularly in low prevalence settings or when mixed strains are present (Van Deun et al., 2013).

Based on the differential non-cross-linking aggregation of Au-nanoprobes, Baptista's group developed a simple and straightforward colorimetric method for Mtb identification and single base mutation discrimination in rpoB (Veigas et al., 2010), which constitutes the first application of AuNPs for the specific detection of RIF resistant Mtb. This approach uses an Au-nanoprobe assay for the rapid detection of MTBC strains and simultaneous characterisation of mutations associated with RIF resistance, namely mutations in codons 516, 526, and 531 of $r p o B$ gene from MTBC clinical specimens with remarkable sensitivity in a few hours. To assure high selectivity and sensitivity, two nanoprobes are simultaneously used to tackle each mutation - one recognizing the wild-type sequence and another for the mutated. By doing so, this approach correctly detected the presence of DNA from members of the MTBC in $83.3 \%$ of all tested samples. The initial approach required a simple PCR amplification of a large region spreading the targets sequences for the nanoprobes, and the resulting amplicons tested directly with the Au-nanoprobe system. The molecular characterisation step takes only 15 min to yield a colorimetric result that, through the use of a suitable photodetector (e.g., UV/visible spectrophotometer, microplate reader, etc.) allows for medium throughput analysis at a peripheral laboratory. A limit of detection could be set at $75 \mathrm{nM}$, however, for robust single base mismatch determination, $117 \mathrm{nM}$ of DNA target were used per assay (see also Table 2).

More recently, the same group extended and improved this detection strategy towards the simultaneous discrimination of specific mutations within inhA and $r p o B$ genes in PCR amplified DNA from isolates. Using a multiplex PCR reaction, it was possible to assess both loci in parallel, and extend the potential of the Au-nanoprobe method to MDRTB molecular characterisation with special application in the most frequent Portuguese genotypes (Pedrosa etal., 2014). Based on the molecular signatures of susceptibility of MTBC members to first line antibiotics, RIF, INH, and ethionamide (ETH), a two-step approach was developed, based on the multi loci PCR amplification of gene fragments and subsequent hybridisation with specific Au-nanoprobe. The two target sequences harbor the most common mutations associated with resistance to these antibiotics, $r p o B$ S531L, inhA C(-)15T and are amplified by a set of $r p o B$ primer pairs flanking unique regions specific for MTBC members - first level of identification. The MTBC Au-nanoprobe constitutes a second level of identification. Another two sets of nanoprobes are used to discriminate the desired mutations. This approach brings new possibilities for MDRTB diagnostics as the Au-nanoprobe methodology may become an useful tool for MDRTB molecular characterisation at a point-of-need (Pedrosa et al., 2014).

Conventional TB diagnosis methods (such as Ziehl-Neelsen or Kinyoun for staining sputum smears, egg-based media for culture, and solid media for antimicrobial susceptibility testing) have been used for almost 50 years presenting low sensitivity, specificity, and a high turn-around time. Although some laboratories use fluorochrome stains and liquid-based media for cultures, small hospitals or clinics cannot use these methods due to the need of high technical expertise, equipment, and expensive materials. The quality of sputum specimens and contamination of specimens due to inappropriate storage and/or long transport times to the laboratory has been a critical bottleneck (Wilson, 2011).

The development of complete, accurate and simple TB diagnostic tests able to target relevant $\mathrm{TB}$ sequences and assessing multidrug resistance cases has been one of the major bottlenecks for TB effective detection and treatment. Au-nanoprobes integrated within a paper-based platform may be proven to be an accurate, rapid, low-cost, and user friendly nanosystem for the identification of specific DNA sequences of TB, confirming infection and allowing identification of MDRTB strains. This nanosystem allows earlier treatment, reduction of time spent 
on inappropriate and ineffective treatment and reduction of MDRTB spread in congregate settings making it ideal for large screening and/or at point-of-need. The ability of coupling the LAMP amplification strategy to specific Au-nanoprobes translates into additional benefits: it eliminates the PCR amplification step bypassing the need of specialized machines and technicians, assessing multiple antibiotic resistances in the field and reaching remote communities.

Integration of isothermal amplification techniques to AuNPs strategies. Isothermal amplification techniques have been recently developed as an alternative to PCR for target DNA amplification and detection without the use of a thermocycler (Gill and Ghaemi, 2008). Thermophilic helicase-dependent isothermal amplification uses a thermostable helicase to unwind the double stranded DNA (dsDNA) and generate single stranded templates that are used for further polymerase amplification (Lixin et al., 2005). The dsDNA separation and amplification are performed at the same temperature, which makes this technique suitable for development of point-of-care microbial detection systems, since a thermocycler is not required for DNA denaturation and amplification (Tomita et al., 2008; Jeong et al., 2009). Recently, a AuNP based DNA biosensor for the detection of Mtb using thermophilic helicase-dependent isothermal amplification was developed (Torres-Chavolla and Alocilja, 2011). In recent years, Loop-mediated isothermal amplification (LAMP, Eiken Chemical Co. Ltd., Tokyo, Japan) assay has been introduced for the diagnosis of pulmonary TB (Yuan et al., 2013). The general LAMP procedure uses four primers to achieve a cyclical amplification process based on spontaneous formation of stem-loop DNA structures. This process also utilizes a polymerase with strand displacement capability. LAMP has been used to successfully detect a wide range of pathogens including malaria, HIV, and multiplexed detection of bacteria (Hartman et al., 2013).

Further improvements to the Au-nanoprobe system described above were attained via LAMP amplification strategy coupled to specific Au-nanoprobes for molecular identification of MTBC members and resistance signatures, such as RIF resistance (Veigas etal., 2013). Taking advantage of such features, they demonstrated that the non-cross-linking system is capable to discriminate the $r p o B$ S531L point mutation on LAMP products and, thus, opening new possibilities for MDRTB diagnostics in remote environments and at a point-of-care. LAMP originates long DNA concatamers that can easily be assessed via a set of nanoprobes for individual sequence identities, demonstrating that it is possible to use an Au-nanoprobe based strategy to detect single point alteration on isothermally amplified DNA products.

Despite the several benefits presented by these nanodiagnostics systems, translation into the clinics is still unaccomplished. Most of the TB nanosystems reported in the literature still lack validation and for most of them integration in one simple platform capable of eliminating the need for DNA purification and amplification is of utmost importance. Refinement of these laboratory strategies into one single nanodevice may speed up translation into the field.
Rolling circle amplification. Following on the development of isothermal amplification methods, Xiang et al. (2013) developed a surface-anchored rolling circle amplification (RCA) integrated with Au-nanoprobes to isothermally detect multiple point mutations associated with MDRTB with a wild-type to mutant ratio of 5000:1. This work introduced a new SPR method for multiplex mutation detection based on surface signal amplification. The high sensitivity and specificity of this method mainly attributed to the high-fidelity of ligation, multiplexing characteristics of probes, amplification potential of surface-anchored RCA and Au NPs, and intrinsically high sensitivity of SPR biosensor. The L-RCA by ligase relies on base pairing principle which requires perfect complementarity on the ligation nick. It not only forbids the mismatch but also has a low occurrence of false positive results when compared to PCR (Lizardi et al., 1998). Because RCA amplifies only the circular PLP without accumulation of target templates over time, it minimizes the risk of contamination and the potential biohazard. Besides, the Au-nanoprobes further enhance identification due to the sandwich hybridisation. Upon recognition, each point mutation is identified by locating into the corresponding channel on a chip, which allows the immobilized primer (capture probe)template (circular PLP) complex to isothermally amplify as RCA and further amplified by AuNPs. Binding of the AuNPs to the RCA products acts as the electromagnetic field coupling to the gold film, thus enhancing the plasmon resonance derived by excitation by the incident light, leading to improvement of the transduction of small changes in refractive index on the chip surface media, thus improving sensitivity (Petryayeva and Krull, 2011).

\section{NANODIAGNOSTICS FOR POINT OF CARE APPLICATIONS}

Despite the amazing advances of nanotechnology the effective translation to the clinical setting and to the molecular detection and/or characterisation has not been fully applied (Hauck et al., 2010). Nanotechnology, and NPs, based molecular identification systems have focused on increasing sensitivity and speed when compared to traditional methodologies. However, nowadays researchers have been gearing their efforts towards the development of nanotechnology-based systems that are affordable, robust and reproducible, making them suitable for applications even in areas that lack dedicated and expensive laboratory equipment. In fact, AuNPs based systems have been proposed and used for the identification of different pathogens with one common ground - making it simple and affordable. Considering that most of these systems rely on the molecular recognition of selective and specific sequences in DNA, we are only one step away from identifying molecular signatures of resistance. In fact, only by bringing together these platforms and those at the forefront of antibiotic resistance characterisation (e.g., microbiologist and clinicians), definite translation can be achieved. Technology integration together with the possibility of miniaturization is of utmost importance for the development of an integrated biosensor suitable for peripheral laboratories and/or point-of-care diagnostics, providing a new tool in the fight against TB.

Nonetheless, there has been some effort towards bringing these technologies to point-of-care application. For example, the $\mathrm{Au}$-nanoprobe system for characterisation of mutations associated to drug resistance in TB has been further integrated with a 
paper-based platform for fast and easy to use detection of MTBC members - Gold on Paper (Veigas et al., 2012b; Costa et al., 2014). Gold on Paper is the working concept of integrating a paper micro well platform and a biomolecular detection scheme based on Aunanoprobes. Gold on Paper showed to be capable of efficiently detect MTBC members directly and, by means of a smartphone device, analyzing data on the spot while maintaining sensitivity and specificity. This demonstrates that systems such as Gold on Paper may be easy to perform without the need for expensive and complex laboratory set up. Using this concept, it is possible to attain a positive identification of the pathogen within one hour, which via the use of a generic "smart" mobile device allows for complete analysis at a peripheral laboratory, and transmit digital information over existing communications channels, combined with GPS location metadata inserted into the captured digital images (Veigas et al., 2012b). The limitation imposed by the DNA sample preparation is greatly overcome by the potential use of this methodology to identify and characterize the molecular signatures involved in antibiotic resistance. This integrated diagnostics scheme can then forward the attained data to a centralized offsite server allowing for monitoring of TB in real-time that could be proven extremely useful in remote areas of the globe lacking resources (Veigas et al., 2012b).

Monitoring for drug-induced liver injury (DILI) via serial transaminase measurements in patients on potentially hepatotoxic medications (e.g., for HIV and TB) is routine in resourcerich nations, but often unavailable in resource-limited settings. Towards enabling universal access to affordable point-of-care screening for DILI, Pollock et al. (2013) have performed the first field evaluation of a paper-based, microfluidic finger-stick test for rapid, semi-quantitative, visual measurement of blood alanine aminotransferase. The objective was to assess operational feasibility, inter-operator variability, lot variability, device failure rate, and accuracy, to inform device modification for further field testing. The paper-based alanine aminotransferase test was performed at point-of-care on fingerstick samples from 600 outpatients receiving HIV treatment in Vietnam (Pollock et al., 2013). This first field study performed with a paper-based microfluidic device opens the door to development of similar assays for other important analytes and also for assessing MDRTB and MRSA.

Based on these principles new technologies were developed and are today available in the market. For example, Nanosphere offers two products approved by the FDA, one aimed at identifying typical mutations in coagulation factors without the need for nucleic acid amplification; another used to genotype polymorphisms associated with warfarin metabolism. In both cases the samples are processed through a cartridge where the sample analyzed via an automated processor and reader (Lefferts et al., 2010; Maurice et al., 2010).

\section{CONCLUSIONS AND FUTURE PERSPECTIVES}

Over the past decades, noble metal NPs, due to their optical and physic-chemical properties, have been used in proof-of-concept biosensing tools for the sensitive detection of pathogens of interest. Amongst these biosensing platforms, several have focused on the specific identification of DNA/RNA sequences associated to molecular signatures of infection and antibiotic resistance. AuNP based assays have progressively been integrated into sensing platforms capable of increasing sensitivity and lowering costs. Here, we provided an overview of existing strategies relying on the use of AuNPs for detection of molecular markers of antibiotic resistance. Despite the desperate need for robust, yet simple and cheap, screening tools to identify MDR pathogens, there are not that many concepts making it through to validation in the laboratory set. It is clear that microbiologists need to integrate the multidisciplinary teams that provide for nanodiagnostics development so as to widen the scope of combinations and modalities that can be easily coupled to current molecular nanodiagnostics technologies so as to facilitate integration to the lab and clinical setting.

Detection strategies based on AuNPs provide comparable detection capability to that of standard techniques but at a fraction of cost and time, usually not requiring cumbersome sample preparation or equipment. As such, nanoparticle based approaches are expected to be incrementally applied to MDR characterisation and pathogen detection with particular emphasis for systems capable to operate at point-of-need. However, despite the massive investment in these technologies, translation to the clinics is yet to be fulfilled. Most of the reported systems in the literature still lack validation and/or are in pre-clinic stages with few commercially available products being available to the clinician. The next step is clearly to focus on the translation of some of the strategies that exist in the lab into the field and to the bedside.

\section{ACKNOWLEDGMENTS}

The authors thank FCT/MEC for financial support through CIGMH (PEst-OE/SAU/UI0009/2011-14); PTDC/BBB-NAN/ 1812/2012 and SFRH/BD/78970/2011 for Bruno Veigas.

\section{REFERENCES}

Abebe, G., Paasch, F., Apers, L., Rigouts, L., and Colebunders, R. (2011). Tuberculosis drug resistance testing by molecular methods: opportunities and challenges in resource limited settings. J. Microbiol. Methods 84, 155-160. doi: 10.1016/j.mimet.2010.11.014

Arbefeville, S. S., Zhang, K., Kroeger, J. S., Howard, W. J., Diekema, D. J., and Richter, S. S. (2011). Prevalence and genetic relatedness of methicillin-susceptible Staphylococcus aureus isolates detected by the Xpert MRSA nasal assay. J. Clin. Microbiol. 49, 2996-2999. doi: 10.1128/JCM.00046-11

Armstead, A. L., and Li, B. (2011). Nanomedicine as an emerging approach against intracellular pathogens. Int. J. Nanomed. 6, 3281-3293. doi: 10.2147/IJN.S27285

Aspler, A., Menzies, D., Oxlade, O., Banda, J., Mwenge, L., Godfrey-Faussett, P., et al. (2008). Cost of tuberculosis diagnosis and treatment from the patient perspective in Lusaka, Zambia. Int. J. Tuberc. Lung Dis. 12, 928-935.

Azzazy, H. M. E., and Mansour, M. M. H. (2009). In vitro diagnostic prospects of nanoparticles. Clin. Chim. Acta 403, 1-8. doi: 10.1016/j.cca.2009.01.016

Azzazy, H. M. E., Mansour, M. M. H., and Kazmierczak, S. C. (2006). Nanodiagnostics: a new frontier for clinical laboratory medicine. Clin. Chem. 52, 1238-1246. doi: 10.1373/clinchem.2006.066654

Azzazy, H. M. E., Mansour, M. M. H., and Kazmierczak, S. C. (2007). From diagnostics to therapy: prospects of quantum dots. Clin. Biochem. 40, 917-927. doi: 10.1016/j.clinbiochem.2007.05.018

Bailey, R. C., Nam, J. M., Mirkin, C. A., and Hupp, J. T. (2003). Real-time multicolor DNA detection with chemoresponsive diffraction gratings and nanoparticle probes. J. Am. Chem. Soc. 125, 13541-13547. doi: 10.1021/ja035479k

Baptista, P. V., Doria, G., Henriques, D., Pereira, E., and Franco, R. (2005). Colorimetric detection of eukaryotic gene expression with DNA-derivatized gold nanoparticles. J. Biotechnol. 119, 111-117. doi: 10.1016/j.jbiotec.2005.02.019

Baptista, P. V., Koziol-Montewka, M., Paluch-Oles, J., Doria, G., and Franco, R. (2006). Gold-nanoparticle-probe-based assay for rapid and direct detection of 
Mycobacterium tuberculosis DNA in clinical samples. Clin. Chem. 52, 1433-1434. doi: 10.1373/clinchem.2005.065391

Baptista, P. V., Pereira, E., Eaton, P., Doria, G., Miranda, A., Gomes, I., et al. (2008). Gold nanoparticles for the development of clinical diagnosis methods. Anal. Bioanal. Chem. 391, 943-950. doi: 10.1007/s00216-007-1768-z

Barnard, M., Albert, H., Coetzee, G., O’Brien, R., and Bosman, M. E. (2008). Rapid molecular screening for multidrug-resistant tuberculosis in a high-volume public health laboratory in South Africa. Am. J. Respir. Crit. Care Med. 177, 787-792. doi: 10.1164/rccm.200709-1436OC

Bischof L. J., Lapsley, L., Fontecchio, K., Jacosalem, D., Young, C., Hankerd, R., et al. (2009). Comparison of chromogenic media to BD GeneOhm methicillinresistant Staphylococcus aureus (MRSA) PCR for detection of MRSA in nasal swabs. J. Clin. Microbiol. 47, 2281-2283. doi: 10.1128/JCM.02256-08

Branton, D., Deamer, D. W., Marziali, A., Bayley, H., Benner, S. A., Butler, T., et al. (2008). The potential and challenges of nanopore sequencing. Nat. Biotechnol. 26, 1146-1153. doi: 10.1038/nbt.1495

Chadwick, S. G., Prasad, A., Smith, W. L., Mordechai, E., Adelson, M. E., and Gygax, S. E. (2013). Detection of epidemic USA300 community-associated methicillinresistant Staphylococcus aureus strains by use of a single allele-specific PCR assay targeting a novel polymorphism of Staphylococcus aureus pbp3. J. Clin. Microbiol. 51, 2541-2550. doi: 10.1128/JCM.00417-13

Chan, W. S., Tang, B. S., Boost, M. V., Chow, C., and Leung, P. H. (2014). Detection of methicillin-resistant Staphylococcus aureus using a gold nanoparticle-based colourimetric polymerase chain reaction assay. Biosens. Bioelectron. 15, 105-111. doi: 10.1016/j.bios.2013.09.027

Chang, Y. C., Yang, C. Y., Sun, R. L., Cheng, Y. F., Kao, W. C., and Yang, P. C. (2013). Rapid single cell detection of Staphylococcus aureus by aptamer-conjugated gold nanoparticles. Sci. Rep. 3, 1863 doi: 10.1038/srep01863

Chastre, J., Blasi, F., Masterton, R. G., Rello, J., Torres, A., and Welte, T. (2014). European perspective and update on the management of nosocomial pneumonia due to methicillin-resistant Staphylococcus aureus after more than 10 years of experience with linezolid. Clin. Microbiol. Infect. 4, 19-36. doi: 10.1111/14690691.12450

Chi, X., Huang, D., Zhao, Z., Zhou, Z., Yin, Z., and Gao, J. (2012). Nanoprobes for in vitro diagnostics of cancer and infectious diseases. Biomaterials 33, 189-206. doi: 10.1016/j.biomaterials.2011.09.032

Costa, M. N., Veigas, B., Jacob, J. M., Santos, D. S., Gomes, J., Baptista, P. V., et al. (2014). A low cost, safe, disposable, rapid and self-sustainable paper-based platform for diagnostic testing: lab-on-paper. Nanotechnology 25, 94006. doi: 10.1088/0957-4484/25/9/094006

Costa, P., Amaro, A., Botelho, A., Inácio, J., and Baptista, P. V. (2010). Gold nanoprobes assay for identification of mycobacteria from the Mycobacterium tuberculosis complex. Clin. Microbiol. Infect. 16, 1464-1469. doi: 10.1111/j.14690691.2010.03120.x

Das, M., Sumana, G., Nagarajan, R., and Malhotra, B. D. (2010). Application of nanostructured $\mathrm{ZnO}$ films for electrochemical DNA biosensor. Thin Solid Films 519, 1196-1201. doi: 10.1016/j.tsf.2010.08.069

Garner, P., Smith, H., Munro, S., and Volmink, J. (2007). Promoting adherence to tuberculosis treatment. Bull. World Health Organ. 85, 404-406. doi: 10.2471/BLT.06.035568

Gaspar, M. M., Cruz, A., Fraga, A. G., Castro, A. G., Cruz, M. E., and Pedrosa, J. (2008). Developments on drug delivery systems for the treatment of mycobacterial infections. Curr. Top. Med. Chem. 8, 579-591. doi: 10.2174/1568026087 83955629

Gill, P., and Ghaemi, A. (2008). Nucleic acid isothermal amplification technologies: a review. Nucleosides Nucleotides Nucleic Acids 27, 224-243. doi: 10.1080/15257770701845204

Halfpenny, K. C., and Wright, D. W. (2010). Nanoparticle detection of respiratory infection, Wiley Interdisciplinary Reviews. Nanomed. Nanobiotechnol. 2, 277290. doi: 10.1002/wnan.83

Hartman, M. R., Ruiz, R. C., Hamada, S., Xu, C., Yancey, K. G., Yu, Y., et al. (2013). Point-of-care nucleic acid detection using nanotechnology Nanoscale 5, 10141-10154. doi: 10.1039/C3NR04015A

Hauck, T. S., Gao, S. G. Y., and Chan, W. C. W. (2010). Nanotechnology diagnostics for infectious diseases prevalent in developing countries. Adv. Drug Deliv. Rev. 62 , 438-448. doi: 10.1016/j.addr.2009.11.015

Jain, K. K. (2005). Nanotechnology in clinical laboratory diagnostics. Clin. Chim. Acta 328, 37-54. doi: 10.1016/j.cccn.2005.03.014
Jain, K. K. (2007). Applications of nanobiotechnology in clinical diagnostics. Clin. Chem. 53, 2002-2009. doi: 10.1373/clinchem.2007.090795

Jeong, Y. J., Park, K., and Kim, D. E. (2009). Isothermal DNA amplification in vitro: the helicase-dependent amplification system. Cell. Mol. Life Sci. 66, 3325-3336. doi: 10.1007/s00018-009-0094-3

Johnson, C. J., Zhukovsky, N., Cass, A. E. G., and Nagy, J. M. (2007). Proteomics, nanotechnology and molecular diagnostics. Proteomics 8, 715-730. doi: 10.1002/pmic.200700665

Jung, Y. L., Jung, C., Parab, H., Li, T., and Park, H. G. (2010). Direct colorimetric diagnosis of pathogen infections by utilizing thiol-labeled PCR primers and unmodified gold nanoparticles. Biosens Bioelectron. 25, 1941-1946. doi: 10.1016/j.bios.2010.01.010

Kaittanis, C., Santra, S., and Perez, J. M. (2010). Emerging nanotechnology-based strategies for the identification of microbial pathogenesis. Adv. Drug Deliv. Rev. 62, 408-423. doi: 10.1016/j.addr.2009.11.013

Kelley, P. G., Grabsch, E. A., Farrell, J., Xie, S., Montgomery, J., Mayall, B., et al. (2011). Evaluation of the Xpert MRSA/SA Blood Culture assay for the detection of Staphylococcus aureus including strains with reduced vancomycin susceptibility from blood culture specimens. Diagn. Microbiol. Infect. Dis. 70, 404-407. doi: 10.1016/j.diagmicrobio.2011.02.006

Larguinho, M., and Baptista, P. V. (2012). Gold and silver nanoparticles for clinical diagnostics - from genomics to proteomics. J. Proteomics 75, 2811-2823. doi: 10.1016/j.jprot.2011.11.007

Lefferts, J. A., Schwab, M. C., Dandamudi, U. B., Lee, H. K., Lewis, L. D., and Tsongalis, G. J. (2010). Warfarin genotyping using three different platforms. Am. J. Transl. Res. 2, 441-446.

Liandris, E., Gazouli, M., Andreadou, M., Comor, M., Abazovic, N., Sechi, L. A., et al. (2009). Direct detection of unamplified DNA from pathogenic mycobacteria using DNA-derivatized gold nanoparticles. J. Microbiol. Methods 78, 260-264. doi: 10.1016/j.mimet.2009.06.009

Lin, C. C., Yang, Y. M., Liao, P. H., Chen, D. W., Lin, H. P., and Chang, H. C. (2014). A filter-likeAuNPs@MS SERS substrate for Staphylococcus aureus detection.Biosens. Bioelectron. 53, 519-527. doi: 10.1016/j.bios.2013.10.017

Liu, W. T. (2006). Nanoparticles and their biological and environmental applications. J. Biosci. Bioeng. 102, 1-7. doi: 10.1263/jbb.102.1

Lixin, A., Tang, W., Ranalli, T. A., Kim, H. J., Wytiaz, J., and Kong, H. (2005). Characterization of a thermostable UvrD helicase and its participation in helicase-dependent amplification. J. Biol. Chem. 280, 28952-28958. doi: 10.1074/jbc.M503096200

Lizardi, P. M., Huang, X., Zhu, Z., Bray-Ward, P., Thomas, D. C., and Ward, D. C. (1998). Mutation detection and single-molecule counting using isothermal rolling-circle amplification. Nat. Genet. 19, 225-232. doi: 10.1038/898

Maurice, C. B., Barua, P. K., Simses, D., Smith, P., Howe, J. G., and Stack, G. (2010). Comparison of assay systems for warfarin-related CYP2C9 and VKORC1 genotyping. Clin. Chim. Acta 411, 947-954. doi: 10.1016/j.cca.2010.03.005

Mirkin, C. A., Letsinger, R. L., Mucic, R. C., and Storhoff, J. J. (1996). A DNAbased method for rationally assembling nanoparticles into macroscopic materials. Nature 15, 607-609. doi: 10.1038/382607a0

Mothershed, E. A., and Whitney, A. M. (2005). Nucleic acid-based methods for the detection of bacterial pathogens: present and future considerations for the clinical laboratory. Clin. Chim. Acta 363, 206-220. doi: 10.1016/j.cccn.2005.05.050

Musser, J. M. (1995). Antimicrobial agent resistance in Mycobacteria: molecular genetic insights. Clin. Microbiol. Rev. 8, 496-514.

Ngo, Y. H., Li, D., Simon, G. P., and Garnier, G. (2011). Paper surfaces functionalized by nanoparticles. Adv. Colloid Interface Sci. 163, 23-38. doi: 10.1016/j.cis.2011.01.004

Pedrosa, P., Veigas, B., Machado, D., Couto, I., Viveiros, M., and Baptista, P. V. (2014). Gold nanoprobes for multi loci assessment of multi-drug resistant tuberculosis. Tuberculosis 94, 332-337. doi: 10.1016/j.tube.2013.12.009

Pelgrift, R. Y., and Friedman, A. J. (2013). Nanotechnology as a therapeutic tool to combat microbial resistance. Adv. Drug Deliv. Rev. 65, 1803-1815. doi: 10.1016/j.addr.2013.07.011

Petryayeva, E., and Krull, U. J. (2011). Localized surface plasmon resonance: nanostructures, bioassays and biosensing-a review. Anal. Chim. Acta 706, 8-24. doi: 10.1016/j.aca.2011.08.020

Pissuwan, D., Cortie, C. H., Valenzuela, S. M., and Cortie, M. B. (2010). Functionalised gold nanoparticles for controlling pathogenic bacteria. Trends Biotechnol. 28, 207-213. doi: 10.1016/j.tibtech.2009.12.004 
Pollock, N. R., McGray, S., Colby, D. J., Noubary, F., Nguyen, H., Nguyen, T. A., etal. (2013). Field evaluation of a prototype paper-based point-of-care fingerstick transaminase test. PLoS ONE 8:e75616. doi: 10.1371/journal.pone. 0075616

Rosi, N. L., and Mirkin, C. A. (2005). Nanostructures in biodiagnostics. Chem. Rev. 105, 1547-1562. doi: 10.1021/cr030067f

Sato, K., Hosokawa, K., and Maeda, M. (2003). Rapid aggregation of gold nanoparticles induced by non-cross-linking DNA hybridization. J. Am. Chem. Soc. 125 8102-8103. doi: 10.1021/ja034876s

Shinde, S. B., Fernandes, C. B., and Patravale, V. B. (2012). Recent trends in in-vitro nanodiagnostics for detection of pathogens. J. Control Release 159, 164-180. doi: 10.1016/j.jconrel.2011.11.033

Soini, H., and Musser, J. M. (2001). Molecular diagnosis of Mycobacteria. Clin. Chem. 47, 809-814.

Soo, P. C., Horng, Y. T., Chang, K. C., Wang, J. Y., Hsueh, P. R.,Chuang, C. Y., etal. (2009). A simple gold nanoparticle probes assay for identification of Mycobacterium tuberculosis and Mycobacterium tuberculosis complex from clinical specimens. Mol. Cell Probes 23, 240-246. doi: 10.1016/j.mcp.2009. 04.006

Storhoff, J. J., Lucas, A. D., Garimella, V., Bao, Y. P., and Müller, U. R. (2004). Homogeneous detection of unamplified genomic DNA sequences based on colorimetric scatter of gold nanoparticle probes. Nat. Biotechnol. 22, 883-887. doi: $10.1038 /$ nbt977

Tallury, P., Malhotra, A., Byrne, L. M., and Santra, S. (2009). Nanobioimaging and sensing of infectious diseases. Adv. Drug Deliv. Rev. 62, 424-437. doi 10.1016/j.addr.2009.11.014

Tomita, N., Mori, Y., Kanda, H., and Notomi, T. (2008). Loop-mediated isothermal amplification (LAMP) of gene sequences and simple visual detection of products. Nat. Protoc. 3, 877-882. doi: 10.1038/nprot.2008.57

Torres-Chavolla, E., and Alocilja, E. C. (2011). Nanoparticle based DNA biosensor for tuberculosis detection using thermophilic helicase-dependent isothermal amplification. Biosens. Bioelectron. 26, 4614-4618. doi: 10.1016/j.bios.2011.04.055

Upadhyayula, V. K. (2012). Functionalized gold nanoparticle supported sensory mechanisms applied in detection of chemical and biological threat agents: a review. Anal. Chim. Acta 715, 1-18. doi: 10.1016/j.aca.2011. 12.008

Van Deun, A., Aung, K. J., Bola, V., Lebeke, R., Hossain, M. A., de Rijk, W. B., etal. (2013). Rifampin drug resistance tests for tuberculosis: challenging the gold standard. J. Clin. Microbiol. 51, 2633-2640. doi: 10.1128/JCM. 00553-13

Veigas, B., Doria, G., and Baptista, P. V. (2012a). Nanodiagnostics for Tuberculosis, Understanding Tuberculosis - Global Experiences and Innovative Approaches to the Diagnosis. Winchester: InTech, 562.
Veigas, B., Jacob, J. M., Costa, M. N., Santos, D. S., Viveiros, M., Inácio, J., et al. (2012b). Gold on paper-paper platform for Au-nanoprobe TB detection. Lab Chip 12, 4802-4808. doi: 10.1039/c2lc40739f

Veigas, B., Machado, D., Perdigão, J., Portugal, I., Couto, I., Viveiros, M., et al. (2010). Au-nanoprobes for detection of SNPs associated with antibiotic resistance in Mycobacterium tuberculosis. Nanotechnology 21, 5101-5108. doi: 10.1088/09574484/21/41/415101

Veigas, B., Pedrosa, P., Couto, I., Viveiros, M., and Baptista, P. V. (2013). Isothermal DNA amplification coupled to Au-nanoprobes for detection of mutations associated to Rifampicin resistance in Mycobacterium tuberculosis. J. Nanobiotechnol. 11, 1-6. doi: 10.1186/1477-3155-11-38

Wang S., Inci, E., Libero, G., Singhal, A., and Demirci, U. (2013). Point-of-care assays for tuberculosis: role of nanotechnology/microfluidics. Biotechnol. Adv. 31, 438-449. doi: 10.1016/j.biotechadv.2013.01.006

Wilson M. L. (2011). Recent advances in the laboratory detection of Mycobacterium tuberculosis complex and drug resistance. Infect. Dis. 52, 1350-1355. doi: $10.1093 / \mathrm{cid} / \mathrm{cir} 146$

World Health Organization [WHO]. (2012). Global Tuberculosis Report 2012. Geneva: WHO.

Xiang, Y., Deng, K., Xia, H., Yao, C., Chen, Q., Zhang, L., et al. (2013). Isothermal detection of multiple point mutations by a surface plasmon resonance biosensor with $\mathrm{Au}$ nanoparticles enhanced surface-anchored rolling circle amplification. Biosens. Bioelectron. 49, 442-449. doi: 10.1016/j.bios.2013.04.044

Yuan, L. Y., Li, Y., Wang, M., Ke, Z. Q., and Xu, W. Z. (2013). Rapid and effective diagnosis of pulmonary tuberculosis with novel and sensitive loop-mediated isothermal amplification (LAMP) assay in clinical samples: a meta-analysis. J. Infect. Chemother. 20, 86-92. doi: 10.1016/j.jiac.2013.07.003

Conflict of Interest Statement: The authors declare that the research was conducted in the absence of any commercial or financial relationships that could be construed as a potential conflict of interest.

Received: 18 July 2014; accepted: 11 August 2014; published online: 28 August 2014. Citation: Veigas B, Fernandes AR and Baptista PV (2014) AuNPs for identification of molecular signatures of resistance. Front. Microbiol. 5:455. doi: 10.3389/fmicb.2014.00455

This article was submitted to Antimicrobials, Resistance and Chemotherapy, a section of the journal Frontiers in Microbiology.

Copyright (c) 2014 Veigas, Fernandes and Baptista. This is an open-access article distributed under the terms of the Creative Commons Attribution License (CC BY). The use, distribution or reproduction in other forums is permitted, provided the original author(s) or licensor are credited and that the original publication in this journal is cited, in accordance with accepted academic practice. No use, distribution or reproduction is permitted which does not comply with these terms. 